\title{
Physical activity, confidence and quality of life among cancer patient-carer dyads
}

\author{
Siu-man Ng ${ }^{1,2^{*}}$, Melody H. Y. Fung ${ }^{1^{*}}$ (D), Jessie S. M. Chan ${ }^{3^{*}}$, Celia H. Y. Chan ${ }^{1}$ and Cecilia L. W. Chan ${ }^{1,2}$
}

\begin{abstract}
Background: Physical activity (PA) has been positively associated with health-related quality of life (HRQoL) among cancer patients and family caregivers. However, there has been no relevant research for patient-caregiver dyads.

Methods: Path analysis, based on the actor-partner interdependence model (APIM), was used to examine the relationship between physical activity and health-related quality of life and explore the mediating role of emotional distress in 233 dyads.

Results: In both patients and caregivers, physical activity had a direct positive effect on physical quality of life (QoL) but not on mental. There was a significant indirect effect of physical activity on health-related quality of life via emotional distress for both dyad members. Patients' and caregivers' confidence in fighting cancer was negatively associated with their own emotional distress. Caregivers' confidence in fighting cancer was positively associated with their physical activity and also negatively associated with patients' emotional distress.

Conclusions: Physical activity may be considered as a possible behavioral and rehabilitation strategy for improving health-related quality of life in patient-caregiver dyads and reducing negative symptoms. Future research and intervention may consider cancer patient-family caregiver dyad as a unit of care.
\end{abstract}

Keywords: Mixed cancer, Patient-caregiver dyads, Physical activity, Quality of life, Mediation, Path analysis

\section{Key point}

- Limited research has addressed the physical activity and health-related quality of life of patient-family caregiver dyads.

- Physical activity could potentially serve as behavioral and rehabilitation strategy for improving healthrelated quality of life in patient-caregiver dyads and reducing negative symptoms.

- Patient-family caregiver dyad may be considered as a unit of care in future research and intervention.

\footnotetext{
* Correspondence: ngsiuman@hku.hk; mhyfung@hku.hk; melodyhyfung@gmail.com; jsmchan9@hku.hk

'Department of Social Work and Social Administration, The University of Hong Kong, 5/F, Jockey Club Tower, The Centennial Campus, Pokfulam, Hong Kong, China

${ }^{3}$ School of Chinese Medicine, Faculty of Medicine, The University of Hong Kong, Pokfulam, Hong Kong, China

Full list of author information is available at the end of the article
}

\section{Background}

Cancer has an adverse effect on health-related quality of life (HRQoL) in patients [1] and their caregivers [2]. Previous studies have associated poor HRQoL with higher levels of stress $[3,4]$, anxiety, and depression $[5,6]$, while physical activity (PA) provides physiological and psychological benefits [7], including reductions in symptoms of depression [8] and emotional distress [9] and improvements in cancer patients' HRQoL during active treatment and rehabilitation $[6,7,10,11]$. A recent review suggested that exercise should be used as an adjunct to standard care [12]. The Clinical Oncology Society of Australia (COSA) also acknowledges the role of physical activity in counteracting adverse effects of cancer and its treatment [13].

Family caregivers have a higher risk of stress, depression, poorer physical health [14], lower psychosocial HRQoL, poorer general health [15], and even higher mortality [16] as compared with the general population.

\section{Springer Open}

(อ) The Author(s). 2021 Open Access This article is licensed under a Creative Commons Attribution 4.0 International License, which permits use, sharing, adaptation, distribution and reproduction in any medium or format, as long as you give appropriate credit to the original author(s) and the source, provide a link to the Creative Commons licence, and indicate if changes were made. The images or other third party material in this article are included in the article's Creative Commons licence, unless indicated otherwise in a credit line to the material. If material is not included in the article's Creative Commons licence and your intended use is not permitted by statutory regulation or exceeds the permitted use, you will need to obtain permission directly from the copyright holder. To view a copy of this licence, visit http://creativecommons.org/licenses/by/4.0/. 
Caregiving may interfere with caregivers' lifestyle and health-promoting behaviors which may result in their insufficient PA [4]. Previous studies have demonstrated lower levels of PA among caregivers than noncaregivers, and those with lower PA levels have more depressive symptoms [17] and worse psychological health [15]. Limited yet important research on PA among family caregivers $[2,18]$ has shown that PA improves caregivers' quality of life (QoL) [2] and reduces their stress, depression, and burden [18].

A meta-analysis concluded interdependence between patients and their caregivers' experiences of psychological distress; family caregivers are negatively affected by the patients' cancer disease while their behavior can in turn influence patient outcomes due to the closeness of the relationship [19]. Reciprocity of emotional wellbeing has been found among patient-caregiver dyads [20], which reflects the importance of mutual support between the caregivers and patients in the recovery process. In addition, caregivers play an important role increasing patients' PA because they act as role models to inspire and increase patients' self-efficacy with respect to PA [21]. Facilitating regular PA among caregivers is therefore beneficial to both caregivers and patients' wellbeing [5]. Given the interdependence of emotional wellbeing as suggested by previous findings, it might be more efficient to deliver information and care to patients-caregiver dyad as a unit instead of intervening individually,

Previous research has led to a model which predicts HRQoL of both cancer patients and their caregivers based on demographic, clinical, and psychosocial characteristics $[22,23]$. These variables include gender, age, socioeconomic status, religious activity, time since diagnosis, therapy status, and confidence in fighting cancer. Other research also demonstrated that PA improved HRQoL of cancer patients $[6,7,10,11]$ and their caregivers [2]; however, there has been no research into the relationships between antecedent factors (person and disease treatment), PA, and HRQoL in cancer patients and their caregivers. While a study of the associations between cancer coping styles and health-related behaviors found that cancer patients with greater "fighting spirit" in their coping reported higher levels of PA [24], it is still unclear whether patients and caregivers' confidence in fighting cancer may also influence their level of PA.

The aim of this study was to investigate the relationship between PA and HRQoL in patient-caregiver dyads through path analysis. We used cross-sectional dyadic data and a modification of the stress-coping model [22]; we assumed that a set of antecedent factors including demographic and clinical characteristics were associated with PA as a coping strategy and influenced HRQoL as the outcome (see Fig. 1). In particular, we hypothesized that PA was both directly and indirectly associated with HRQoL through emotional distress as a potential mediator [10].

\section{Methods \\ Design and procedures}

Here we report baseline data from a randomized controlled trial that examined the effects of Qigong exercise on health-related outcomes in Hong Kong, in people with mixed cancer and their caregivers. We recruited participants through adverts in the mass media. The inclusion criteria were (1) caregiver had no current diagnosis of cancer, (2) patient at their post-treatment stage with screened physical ability to stand for $30 \mathrm{~min}$ of physical exercise, and (3) patient and caregiver had to participate as a pair. The exclusion criteria for both dyad members were as follows: (1) physical impairment which restricted body movement, (2) cognitive impairment which prevented understanding of instructions, (3) comorbid major psychiatric illness other than depression or anxiety, and (4) pregnancy. Two hundred and thirtyeight patient-caregiver dyads gave informed consents and completed the questionnaires. We excluded five pairs because of problems with completed questionnaires (incorrect age data $n=1$; missing caregiver data $n$ $=2$; missing patient data $n=2$ ) yielding a final sample of 233 patient-caregiver dyads. All of the procedures were approved by the institutional review board of the University of Hong Kong.

\section{Measures}

Physical activity was assessed using the International Physical Activity Questionnaire - short form [25]. This nine-item instrument captures self-reported physical activity in the week before completion. Responses are converted to Metabolic Equivalent Task minutes per week (MET-min/week) by multiplying the total number of minutes spent in vigorous activity, moderate intensity activity, and walking over the week by 8.0, 4.0, and 3.3, respectively. The MET scores for each activity level are summed to give an indication of overall physical activity.

Emotional distress was assessed as a latent construct using validated measures of perceived stress, anxiety, and depression as indicators. Perceived stress was measured using the ten-item Chinese Perceived Stress Scale [26]. Responses are given using a five-point scale, total scores range from 0 to 40 and high scores indicate high stress levels. Anxiety and depression were measured using the Chinese Hospital Anxiety and Depression Scale (HADS) [27], a fourteen-item instrument that captures the severity of anxiety and depressive symptoms using a four-point response scale. Total scores for anxiety (7 items) and depression ( 7 items) range from 0 to 21 , higher scores denote more severe symptoms. 


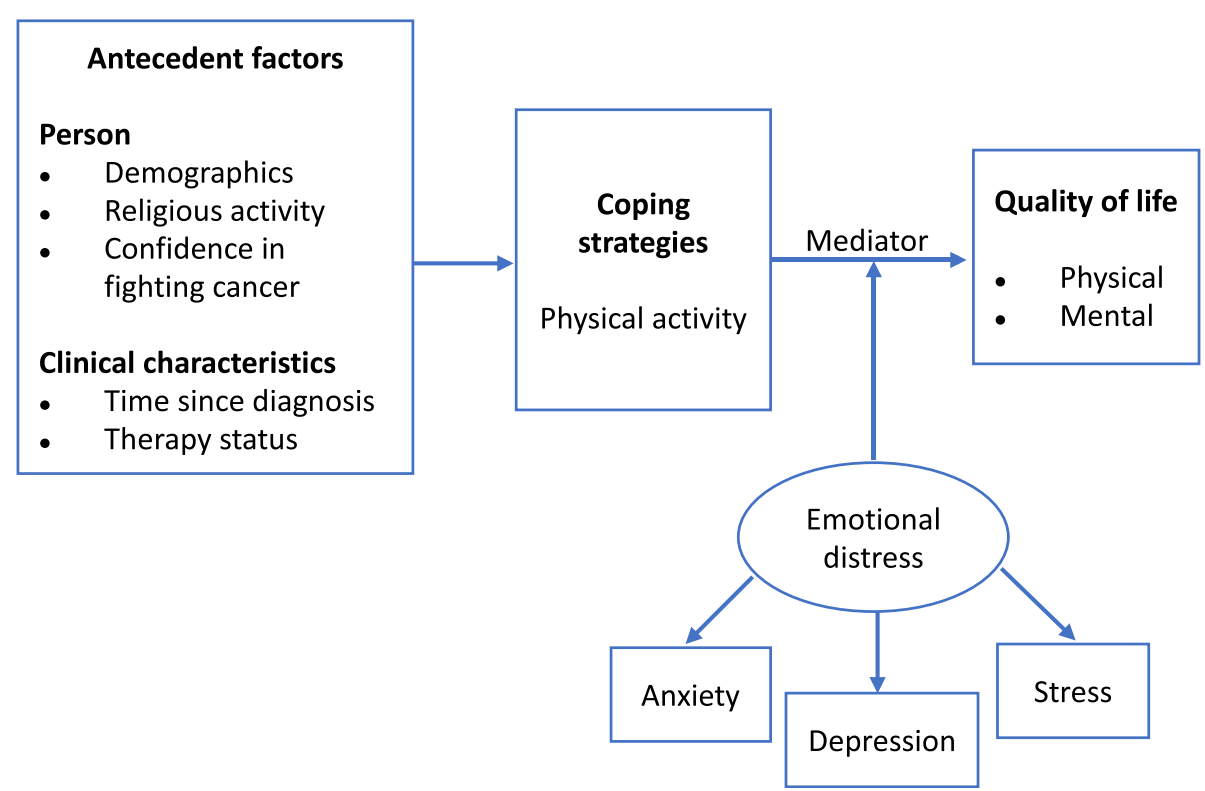

Fig. 1 Conceptual model

QoL was assessed using the Chinese short-form (SF)12 questionnaire $[28,29]$. This twelve-item health survey measures physical and mental QoL using separate 0-100 subscales, higher scores indicate better QoL. In this study, all measurement scales had satisfactory reliability with Cronbach's $\alpha$ exceeding .70 in both patient and caregiver samples.

We used a single item to assess patients' and caregivers' confidence in fighting cancer. The item was selfconstructed by the research team.

\section{Data analysis}

Path analysis was conducted with Mplus version 7.11 [30] using maximum likelihood estimation. Missing data were handled with full information maximum likelihood [31] under the missing at random assumption. Analysis of dyadic data is relatively complex because of the dependence that arises from shared characteristics and experiences; failure to account for this would result in underestimation of the standard errors and bias the results. We used the Actor-Partner Interdependence Model (APIM) [32] to handle interdependence. The APIM model models the responses of both dyad members simultaneously and allows variable interactions between them whilst specifying correlations for all pairs of variables across the dyad. This model not only estimated the conventional actor effects for both the patients and caregivers, but also patient-partner effects (patient predictors influencing caregiver outcomes) and caregiverpartner effects (caregiver predictors influencing patient outcomes).
The proposed path model included covariates (gender, age, socioeconomic status, religious activity, time since diagnosis, therapy status, and confidence in fighting cancer), PA as the predictor, emotional distress as the latent mediator, and physical and mental QoL as outcome variables. We evaluated the direct effect of PA on QoL and the indirect effect of PA on QoL via emotional distress. Model fit was assessed using the following fit indices [33]: comparative fit index $(\mathrm{CFI}) \geq .95$; Tucker-Lewis index (TLI) $\geq .95$; root mean square error of approximation (RMSEA) $\leq .06$ and 90\% confidence interval (C.I.); and standardized root mean square residual (SRMR) $\leq$ .06. To allow for potential non-normal distribution of the indirect effects, bootstrapping with 5000 re-samples was used to produce bootstrap asymmetric 95\% CI [34]. $R$ squared values were computed for the study variables to determine the amount of variance accounted for by the model.

\section{Results}

\section{Participants and descriptive statistics}

The patient sample consisted of cancer patients who had been diagnosed with lung cancer $(24.0 \%)$, breast cancer (19.3\%), colorectal cancer (14.2\%), female reproductive cancers (cervical cancer, ovarian cancer, or uterine cancer; 7.3\%), nasopharyngeal cancer (6.4\%), esophageal and stomach cancer $(3.0 \%)$, prostate cancer $(2.6 \%)$, nonHodgkin's lymphoma (2.6\%), thyroid cancer (1.7\%), and other types of cancer (18.0\%). Table 1 shows the demographic profile of the 233 patient-caregiver dyads. Both samples had a similar demographic profile, with the majority being female, married, and of high socioeconomic 
Table 1 Demographic profile for patients and caregivers

\begin{tabular}{|c|c|c|}
\hline$N=233$ & $\begin{array}{l}\text { Patients } \\
N(\%)\end{array}$ & $\begin{array}{l}\text { Carers } \\
N(\%)\end{array}$ \\
\hline \multicolumn{3}{|l|}{ Gender } \\
\hline Female & $138(59.2)$ & $146(62.7)$ \\
\hline \multicolumn{3}{|l|}{ Education level } \\
\hline Primary or less & $57(24.6)$ & $37(16.0)$ \\
\hline Secondary & $107(46.3)$ & $106(45.9)$ \\
\hline Tertiary & $67(29.0)$ & $88(38.1)$ \\
\hline \multicolumn{3}{|l|}{ Marital status } \\
\hline Married & $194(83.3)$ & $198(85.0)$ \\
\hline \multicolumn{3}{|l|}{ Religious activity } \\
\hline Yes & $119(51.1)$ & $104(44.6)$ \\
\hline \multicolumn{3}{|l|}{ Socioeconomic status } \\
\hline High & $122(52.4)$ & $125(53.6)$ \\
\hline \multicolumn{3}{|c|}{ Caregiver's relationship to cancer patient } \\
\hline Spouse & $146(62.7 \%)$ & \\
\hline Parent & $11(4.7 \%)$ & \\
\hline Child & $31(13.3 \%)$ & \\
\hline \multirow[t]{2}{*}{ Others } & $45(19.3 \%)$ & \\
\hline & $\begin{array}{l}M(S D) \\
\text { Median (interquartile) }\end{array}$ & $M(S D)$ \\
\hline Age (years) & $57.4(10.4)$ & $53.6(12.7)$ \\
\hline Time since diagnosis (years) & $1.0(1.0-2.0)$ & / \\
\hline \multicolumn{3}{|l|}{ Cancer stage } \\
\hline 0 & $8(3.4 \%)$ & \\
\hline 1 & $43(18.5 \%)$ & \\
\hline 2 & $39(16.7 \%)$ & \\
\hline 3 & $70(30.0 \%)$ & \\
\hline 4 & $73(31.3 \%)$ & \\
\hline \multicolumn{3}{|l|}{ Therapy status } \\
\hline Completed & $92(39.5 \%)$ & \\
\hline In therapy & $110(47.2 \%)$ & \\
\hline Waiting for therapy & $31(13.3 \%)$ & \\
\hline Number of therapies & $2.0(1.1)$ & / \\
\hline Confidence in fighting cancer & $3.5(1.0)$ & $3.5(1.0)$ \\
\hline
\end{tabular}

status. Accommodation is taken as a rough indicator of socioeconomic status in Hong Kong [35]. Participants who own or rent a private housing are supposed to be relatively wealthy and therefore classified as having high socioeconomic status. Spouses made up the majority of caregiver (62.7\%).

The mean ages of patients and caregivers were 57.4 years $(S D=10.4)$ and 53.6 years $(S D=12.7)$, respectively. The most common cancer stage in patients was stage $4(31.3 \%)$, followed by stage $3(30.0 \%)$, stage 1 (18.5\%), stage $2(16.7 \%)$, and finally stage $0(3.4 \%)$. The majority $(60.5 \%)$ of the patients were in therapy or waiting for therapy. The median time since diagnosis was 1 year (interquartile range: $1-2$ years) and patients were receiving an average $2.0(S D=1.1)$ types of therapy at the time of the study. Confidence in fighting cancer was expressed using a five-point scale ranging from 1 (not at all confident) to 5 (very confident), and the mean score for both patients and caregivers was $3.5(S D=1.0)$.

Table 2 displays the descriptive statistics for study variables; the scores of members of a dyad were positively correlated with respect to PA, perceived stress, anxiety and depression, and mental QoL but not physical QoL. The patients reported higher perceived stress, anxiety, and depression and lower PA and physical and mental QoL than the caregivers. Using a threshold score of 8 [36], $61.8 \%$ and $56.2 \%$ of the patients were classified as potentially suffering from clinically significant anxiety and depression, respectively. In caregivers, the corresponding figures were $51.1 \%$ and $37.8 \%$ for anxiety and depression, respectively. Table 3 shows the bivariate associations between PA and other study variables separately for cancer patients and family caregivers.

\section{Path model results}

The path model displayed an acceptable fit to the data $(\mathrm{CFI}=.97$; TLI $=.96$; $\mathrm{RMSEA}=.036,90 \% \mathrm{CI}: .020-.049$; SRMR $=.045)$. Detailed results are presented for patients and caregivers in Figs. 2 and 3, respectively. The model explained $6.6 \%$ and $11.4 \%$ of the variance in PA in patients and their caregivers, respectively. Women reported lower levels of PA both as patients $(\beta=-0.43, p<.01)$ and as caregivers $(\beta=-0.48, p<.01)$. In caregivers, PA was positively associated with confidence in fighting cancer $(\beta=0.24, p<.05)$. Caregivers with high socioeconomic status reported lower level of PA $(\beta=-0.24, p<$ $.05)$.

\section{Patients' quality of life}

The model explained $29.5 \%$ of the variance in patients' physical QoL and $65.9 \%$ of the variance in their mental QoL. In patients, PA had a direct positive effect on physical QoL $(\beta=1.99, p<.01)$ but not on mental QoL $(\beta=$ $0.42, p=.34)$. Emotional distress had negative effects on both physical QoL $(\beta=-0.95, p<.01)$ and mental QoL $(\beta=-2.32, p<.01)$. PA had indirect positive effects on physical QoL $(\alpha \beta=0.61, p<.01)$ and mental QoL $(\alpha \beta=$ $1.48, p<.01$ ) via emotional distress. In patients, PA was positively linked with QoL via several steps including a negative relationship between PA and emotional distress, and then a relationship between emotional distress and physical/mental QoL, suggesting that patients with higher level of PA had lower level of emotional distress, which led to higher levels of QoL. 
Table 2 Descriptive statistics of study variables for patients and caregivers $(N=233)$

\begin{tabular}{lllll}
\hline & Patients & Carers & & Correlation coefficient $\boldsymbol{r}$ \\
\hline Physical activity (In) & M (SD) & $\boldsymbol{M}($ SD) & $3.49^{* *}$ & $0.156^{*}$ \\
Perceived stress & $7.3(1.1)$ & $7.6(1.2)$ & $2.47^{*}$ & $0.385^{* *}$ \\
Anxiety & $20.6(4.8)$ & $19.7(5.0)$ & $4.47^{* *}$ & $0.407^{* *}$ \\
Depression & $8.8(4.1)$ & $7.6(3.7)$ & $7.83^{* *}$ & $0.424^{* *}$ \\
Physical quality of life & $8.4(4.3)$ & $6.2(3.8)$ & $10.7^{* *}$ & 0.088 \\
Mental quality of life & $37.2(8.2)$ & $45.2(8.7)$ & $4.78^{* *}$ & $0.249^{* *}$ \\
\hline
\end{tabular}

$t$ test for comparison between cancer patients and caregivers

${ }^{*} p<0.05 ;{ }^{* *} p<0.01$

\section{Patients' emotional distress}

The model explained $27.1 \%$ of the variance in patients' emotional distress. PA had a direct negative effect on emotional distress $(\beta=-0.64, p<.01)$. Higher levels of emotional distress were reported by female patients $(\beta=$ 1.07, $p<.05)$, patients with less confidence in fighting cancer $(\beta=-0.94, p<.01)$, and patients who had caregivers with less confidence in fighting cancer $(\beta=-0.76$, $p<.05)$.

\section{Caregivers' quality of life}

The model explained $24.2 \%$ of the variance in caregivers' physical QoL and 60.6\% of the variance in their mental QoL. In caregivers, PA had a direct positive effect on physical QoL $(\beta=0.87, p<.05)$ but not on mental QoL $(\beta=-0.15, p=.68)$. Emotional distress had negative effects on both physical QoL $(\beta=-0.94, p<.01)$ and mental QoL $(\beta=-2.37, p<.01)$. PA had indirect effects

Table 3 Correlation coefficients among measures on physical activity, emotional distress, and quality of life for cancer patients and caregivers

\begin{tabular}{|c|c|c|c|c|c|}
\hline \multicolumn{6}{|c|}{ For cancer patients } \\
\hline & PA & PSS & Anxiety & Depression & P-QoL \\
\hline PA & 1 & & & & \\
\hline PSS & -.096 & 1 & & & \\
\hline Anxiety & $-.173^{*}$ & $.740^{* * *}$ & 1 & & \\
\hline Depression & $-.172^{*}$ & $.632^{* * *}$ & $.784^{* * *}$ & 1 & \\
\hline P-QoL & $.226^{* *}$ & $-.335^{* * *}$ & $-.355^{* * *}$ & $-.465^{* * *}$ & 1 \\
\hline M-QoL & $.217^{* *}$ & $-.579^{* * *}$ & $-.736^{* * *}$ & $-.727^{* * *}$ & $.215^{* *}$ \\
\hline \multicolumn{6}{|c|}{ For family caregivers } \\
\hline & PA & PSS & Anxiety & Depression & P-QoL \\
\hline PA & 1 & & & & \\
\hline PSS & -.090 & 1 & & & \\
\hline Anxiety & $-.158^{*}$ & $.723^{* * *}$ & 1 & & \\
\hline Depression & $-.188^{* *}$ & $.590^{* * *}$ & $.746^{* * *}$ & 1 & \\
\hline P-QoL & $.176^{* *}$ & $-.264^{* * *}$ & $-.293^{* * *}$ & $-.424^{* * *}$ & 1 \\
\hline M-QoL & $.157^{*}$ & $-.659^{* * *}$ & $-.685^{* * *}$ & $-.657^{* * *}$ & $.190^{* *}$ \\
\hline
\end{tabular}

on physical QoL $(\alpha \beta=0.45, p<.01)$ and mental QoL $(\alpha \beta=1.14, p<.01)$ via emotional distress. Older caregivers reported lower physical QoL $(\beta=-0.19, p<.01)$ but better mental QoL $(\beta=0.10, p<.01)$.

\section{Caregivers' emotional distress}

The model explained $21.5 \%$ of the variance in caregivers' emotional distress. PA had a negative effect on caregivers' emotional distress $(\beta=-0.48, p<.01)$. Caregivers with less confidence in fighting cancer $(\beta=-1.29$, $p<.01)$ reported higher emotional distress.

\section{Mediating effects}

In cancer patients, emotional distress mediated the association between their confidence in fighting cancer and their physical QoL $(\alpha \beta=0.89$, bootstrap 95\% CI: $0.36-$ $1.65, p<.01)$. The patient's emotional distress also mediated the relationship between a caregiver' confidence in fighting cancer and the patient's physical QoL $(\alpha \beta=$ 0.72, bootstrap 95\% CI: $0.10-1.54, p<.05)$. Patients' emotional distress mediated the association between their confidence in fighting cancer and their mental QoL $(\alpha \beta=2.18$, bootstrap 95\% CI: $0.86-3.61, p<.01)$. The patient's emotional distress also mediated the relationship between a caregiver's confidence in fighting cancer and the patient's mental QoL $(\alpha \beta=1.76$, bootstrap 95\% CI: $0.16-3.28, p<.05)$.

Caregivers' confidence in fighting cancer had indirect effects on their physical QoL via PA $(\alpha \beta=0.21$, bootstrap 95\% CI: $0.02-0.55, p<.05)$, via emotional distress $(\alpha \beta=$ 1.21, bootstrap 95\% CI: $0.75-1.87, p<.01$ ), and via PA and emotional distress $(\alpha \beta \gamma=0.11$, bootstrap 95\% CI: $0.02-0.29, p<.01$ ). Similarly, caregivers' confidence in fighting cancer had indirect effects on their mental QoL via emotional distress $(\alpha \beta=3.04$, bootstrap 95\% CI: $1.94-$ $4.26, p<.01)$ and via PA and emotional distress $(\alpha \beta \gamma=$ 0.27 , bootstrap 95\% CI: $0.06-0.65, p<.01$ ).

\section{Discussion}

To the best of our knowledge, this is the first study to examine how PA is related to QoL in cancer patient- 


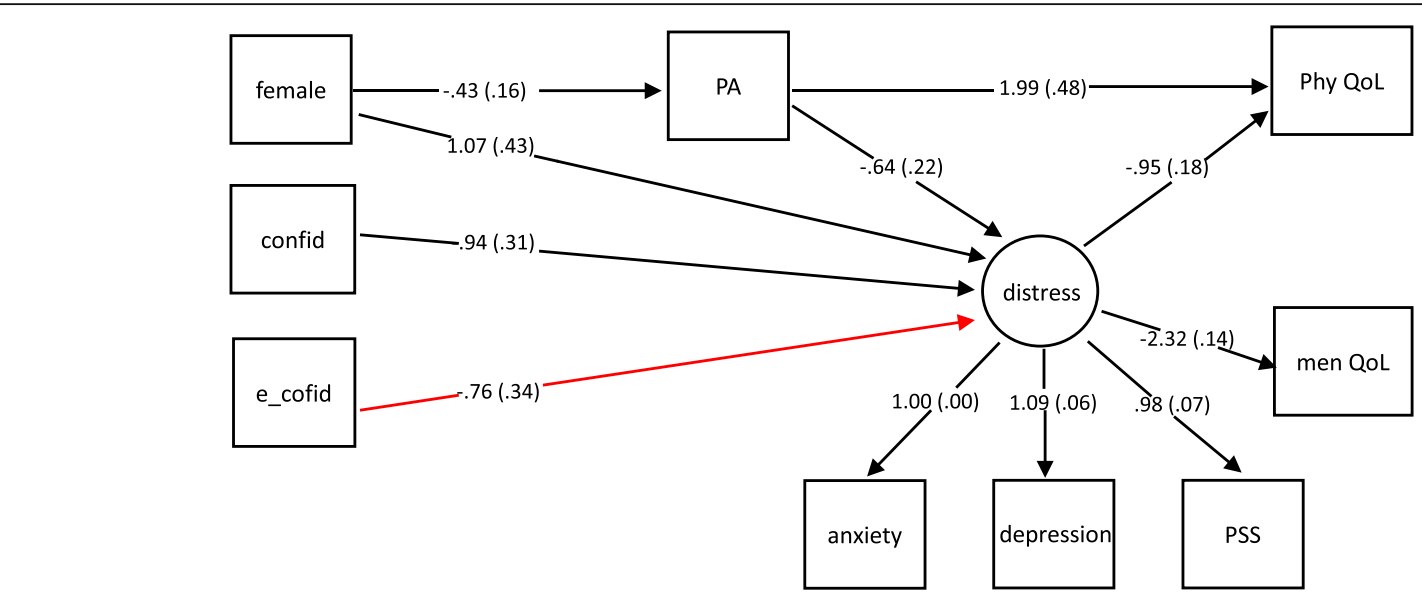

Fig. 2 Path model on the relationships between PA and QoL via emotional distress for patients. Note: All paths coefficients shown are significant with $p<.05$ and refer to actor effects, except the partner effect from caregivers' confidence in fighting cancer to patients' emotional distress (red and bolded arrow); confid = confidence in fighting cancer; PA = physical activity; PSS = perceived stress; phy QoL = physical quality of life; men $\mathrm{QoL}=$ mental quality of life

caregiver dyads. We developed a path model of the associations between PA and HRQoL that indicated positive correlations between the members of a dyad with respect to mental QoL, PA, perceived stress, anxiety, and depression. These findings are in line with results of Kershaw et al. [21] regarding positive correlations between the members of a dyad with respect to mental QoL, but not physical QoL in a sample of 121 prostate cancer patients-spouse dyads. These results suggest that clinicians should consider the care requirements of both members of the cancer patientfamily caregiver dyad [18].

Our study demonstrated that PA has direct or indirect positive effects on HRQoL for both cancer patients and family caregivers, which is consistent with previous systematic reviews $[7,11,37]$. However, few studies have investigated the role of $\mathrm{PA}$ in determining the HRQoL of the family caregivers of cancer patients [18]. This study has showed that PA was positively associated with physical and mental QoL in cancer patient-family caregiver dyads. Unlike findings from a previous populationbased study among Iranian adult population which indicated higher level of PA among people with high SES [38], our findings indicate association of caregivers with high SES with low PA. Further research may be conducted to investigate barriers or concerns among caregivers with high SES in performing PA.

Among patients, PA had direct effects on emotional distress and physical QoL but not on mental QoL. Physical QoL was positively associated with PA, while both physical and mental Qol were negatively associated with emotional distress. Emotional distress mediated the

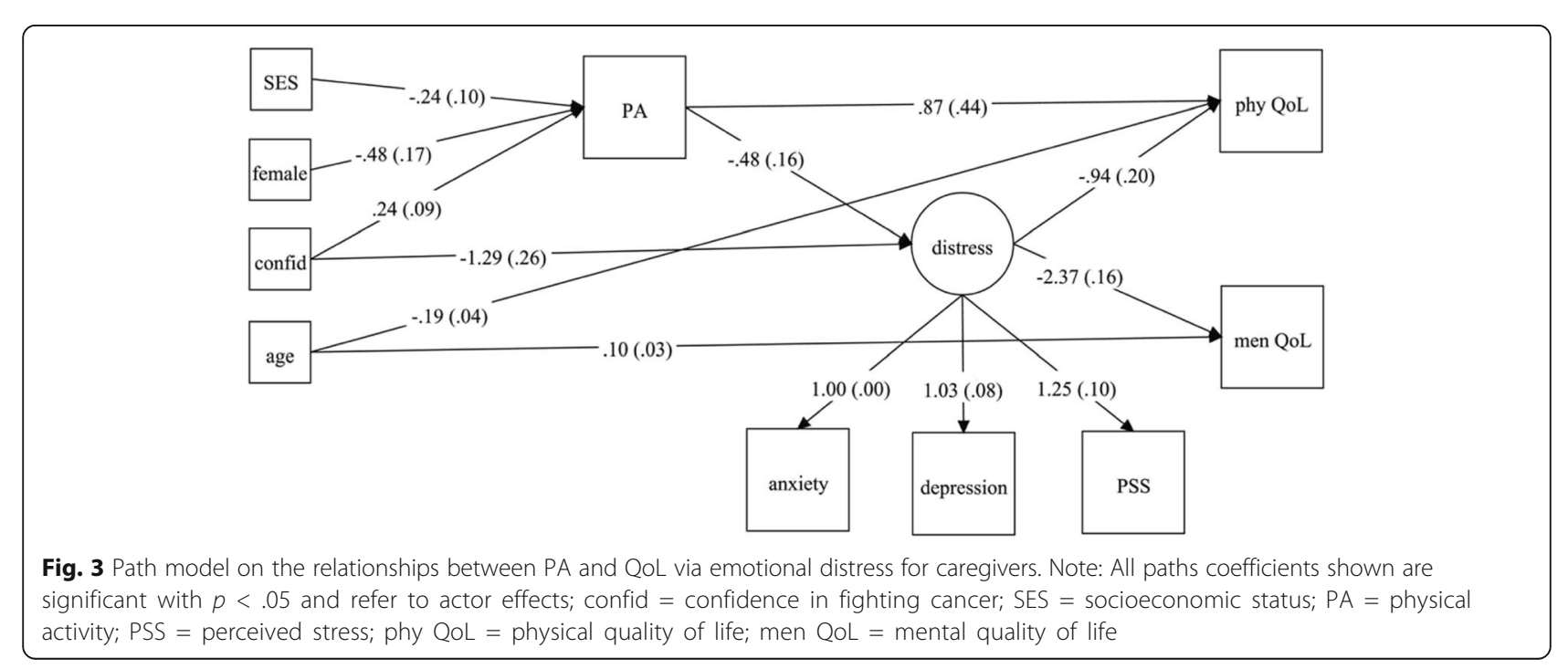


association between PA and physical QoL. The results are in line with findings of a large cohort study of colorectal cancer survivors $(n=1371)$ using multiple linear regression [10]. In our sample, the relationship between PA and mental QoL was also entirely indirect, whereas the relationship between PA and physical QoL was both direct and indirect, which is consistent with the findings of a previous study of patients with multiple sclerosis [39].

In family caregivers, PA directly influenced physical QoL and emotional distress and indirectly affected mental QoL via emotional distress, which is in line with a review of PA intervention on stress and depression among caregivers of people with dementia [18]. Emotional distress was also found to mediate the association between PA and physical QoL. Their age was negatively associated with physical QoL and positively associated with mental QoL, which are consistent with previous research [22].

We assessed confidence in fighting cancer in both cancer patients and their family caregivers; it can be considered a similar construct to the form of self-efficacy used in other studies [22]. Self-efficacy of cancer patients is defined in terms of confidence in managing stress and the changes associated with cancer or treatment, whereas that of family caregivers is defined in terms of confidence in managing the cancer as a caregiver. In this study, confidence in fighting cancer was positively associated with PA in caregivers but not patients. It was negatively associated with emotional distress in both cancer patients and family caregivers. The finding is consistent with a previous study of a relatively small sample of colorectal cancer survivors $(n=62)$ and their family caregivers $(n=42)$ [40]. We also found that confidence in fighting cancer had indirect positive effects on HRQoL via emotional distress in the dyads. These results suggest that confidence in fighting cancer or selfefficacy should be assessed as part of the clinical evaluation of both patients and caregivers [22]. Both PA and confidence in fighting cancer/self-efficacy could be important targets for behavioral and self-management interventions aimed at improving HRQoL [41]. A previous study suggested that family caregivers' social support is essential to cancer patients' participation in PA [21]. Interestingly, although we did not find an association between a caregiver's confidence in fighting cancer and the patient's PA, we found that family caregiver's confidence in fighting cancer was negatively associated with the cancer patient's emotional distress. This implies that a family caregiver's confidence in fighting cancer plays an important role in determining a cancer patient's emotional distress and HRQoL, supporting the argument that family caregivers should be involved in intervention designed to reduce cancer patients' emotional distress and improve their HRQoL [19].

\section{Implications}

This study has a number of clinical implications. First, the positive association between PA and HRQoL in cancer patient-family caregiver dyads suggests that healthcare professionals should use PA as part of interventions aimed at improving the HRQoL of such dyads. Results also suggest that healthcare providers should consider screening individuals to identify those with low confidence in fighting cancer.

Levels of PA were lower in female patients and caregivers than in their male counterparts, which is consistent with recent studies of students and colorectal cancer patients [42]. These results suggest that women need more encouragement to engage in PA when they are cancer patients or caregivers for a patient with cancer. In line with previous research [19], female cancer patients were more likely to report significant emotional distress than male cancer patients. Furthermore, the physical condition of patients with various types of cancer may differ and thus affect their ability and confidence of performing PA. Patients who need additional assistance on recovery from surgeries and their caregivers who have to pay extra effort in taking care of the patients may be more reluctant to spend time on doing PA. Healthcare providers could apply findings of this study to identify high-risk individuals in terms of PA and confidence in fighting cancer.

\section{Limitations}

First, the self-selection recruitment method may have resulted in selection bias. These findings may not generalize to patients whose spouse or other family caregiver is not willing to participate in research of this kind. Second, the sample was predominantly female, welleducated, and married. It is important to determine whether the model presented here is applicable to cancer patients with a different demographic profile. Third, the use of self-report measures was subject to common method bias, which may have inflated correlations among the study variables. Future studies could assess the effects of PA on objectively measurable psychophysiological outcomes. Fourth, we relied on cross-sectional data, which precludes inferences about the causal relationships among the variables. Longitudinal research investigating the impact of changes in PA and emotional distress on HRQoL is needed in the future. Fifth, confidence in fighting cancer was assessed using a single question devised by the research team; this indicator was shown to be associated with PA in family caregivers and with emotional distress in both cancer patients and family caregivers. In future research, it would be advisable to use a validated scale for measuring self-efficacy, self-esteem, or sense of hopelessness instead. 


\section{Conclusions}

This study is the first to investigate the role of PA in determining HRQoL in a mixed sample of 233 cancer patientfamily caregiver dyads. Notwithstanding the limitations, it shows the importance of PA and confidence in fighting cancer to HRQoL in such dyads and supports the development of activity or exercise interventions as a strategy for improving HRQoL in patients and caregivers throughout the course of the disease. It also suggests how healthcare providers could identify individuals who likely to have low levels of PA in order to provide appropriate interventions.

\section{Abbreviations \\ PA: Physical activity; HRQoL: Health-related Quality of Life; APIM: Actor- partner interdependence model; QoL: Quality of life}

\section{Acknowledgements}

The authors thank T.C.T. Fong, L.P. Yuen, their colleagues in the Centre on Behavioral Health, the International Association for Health and Yangsheng and Hong Kong Anti-Cancer Society, as well as the volunteers and all participants who made this study possible. The results of the study are presented clearly, honestly, and without fabrication, falsification, or inappropriate data manipulation.

\section{Authors' contributions}

All authors contributed to the study conception and design. Material preparation, data collection, and analysis were performed by SN, JSMC, CHYC, and CLWC. The first draft of the manuscript was written by JSMC, and all authors commented on previous versions of the manuscript. The authors read and approved the final manuscript.

\section{Funding}

The study was funded by the Behavioral Health Research Fund of the University of Hong Kong.

\section{Availability of data and materials}

The datasets used and analysed during the current study are available from the corresponding author on reasonable request

\section{Declarations}

\section{Ethics approval and consent to participate}

All patient-caregiver dyads gave informed consents to participate in the study. The research was performed in accordance with the standards of ethics outlined in the Declaration of Helsinki and was approved by the Human Research Ethics Committee (HREC) of the University of Hong Kong. (Approval number: EA020312)

\section{Consent for publication}

The manuscript does not contain any individual person's data.

\section{Competing interests}

The authors, Siu-man Ng, Melody Fung, Jessie Chan, Celia Chan, and Cecilia Chan, declare that they have no competing interests.

\section{Author details}

${ }^{1}$ Department of Social Work and Social Administration, The University of Hong Kong, 5/F, Jockey Club Tower, The Centennial Campus, Pokfulam, Hong Kong, China. ${ }^{2}$ Centre on Behavioral Health, The University of Hong Kong, Pokfulam, Hong Kong, China. ${ }^{3}$ School of Chinese Medicine, Faculty of Medicine, The University of Hong Kong, Pokfulam, Hong Kong, China.

Received: 2 June 2020 Accepted: 1 June 2021

Published online: 01 July 2021

\section{References}

1. Richardson LCet al. (2008) Health-related quality of life in cancer survivors between ages 20 and 64 years: population-based estimates from the behavioral risk factor surveillance system. Cancer, 112 6: 1380-1389. https://doi.org/10.1002/cncr.23291

2. Klassen AF, Klaassen R, Dix D, Pritchard S, Yanofsky R, O'Donnell M, Scott A, Sung $L$ (2008) Impact of caring for a child with cancer on parents' healthrelated quality of life. J Clin Oncol, 26 36:5884-5889 https://doi.org/10.1200/ JCO.2007.15.2835

3. Bevans M, Sternberg EM (2012) Caregiving burden, stress, and health effects among family caregivers of adult cancer patients. JAMA, 307:398-403. https://doi.org/10.1001/jama.2012.29, 4.

4. Beesley VL, MA Price, Webb PM (2011) Loss of lifestyle: health behaviour and weight changes after becoming a caregiver of a family member diagnosed with ovarian cancer. Support Care Cancer, 19 12:1949-1956. https://doi.org/10.1007/s00520-010-1035-2.

5. Cameron Jl, Franche RL, Cheung AM, Stewart DE. Lifestyle interference and emotional distress in family caregivers of advanced cancer patients. Cancer. 2002;94(2):521-7. https://doi.org/10.1002/cncr.10212.

6. Sammut L, Ward M, Patel N (2014) Physical activity and quality of life in head and neck cancer survivors: a literature review. Int J Sports Med, 35 09: 794-799. https://doi.org/10.1055/s-0033-1363984.

7. Spence RR, Heesch KC, Brown WJ (2010) Exercise and cancer rehabilitation: a systematic review. Cancer Treat Rev, 36:185-194. https://doi.org/10.1016/j. ctrv.2009.11.003, 2

8. Craft LL, Vanlterson EH, Helenowski IB, Rademaker AW, Courneya KS. Exercise effects on depressive symptoms in cancer survivors: a systematic review and meta-analysis. Cancer Epidemiol Biomark Prev. 2012;21(1):3-19. https://doi.org/10.1158/1055-9965.EPI-11-0634

9. Wenzel JA, Griffith KA, Shang JJ, Thompson CB, Hedlin H, Stewart KJ, DeWeese T, Mock V (2013) Impact of a home-based walking intervention on outcomes of sleep quality, emotional distress, and fatigue in patients undergoing treatment for solid tumors. Oncologist, 18 4:476-484. https:// doi.org/10.1634/theoncologist.2012-0278.

10. Buffart LM, Thong MSY, Schep G, Chinapaw MJM, Brug J, van de Poll-Franse LV (2012) Self-reported physical activity: its correlates and relationship with health-related quality of life in a large cohort of colorectal cancer survivors. PLoS One, 7 5:36164. https://doi.org/10.1371/journal.pone.0036164.

11. Mishra SI et al (2012) Exercise interventions on health-related quality of life for cancer survivors. Cochrane Database Syst Rev https://doi.org/10.1002/14 651858.CD007566.pub2.

12. Gerritsen JK, Vincent AJ (2015) Exercise improves quality of life in patients with cancer: a systematic review and meta-analysis of randomised controlled trials. Br J Sports Med 50, 13, 796, 803 https://doi.org/10.1136/ bjsports-2015-094787.

13. Cormie et al. (2018). Clinical Oncology Society of Australia position statement on exercise in cancer care, 209:184-7.

14. Oyebode J. Assessment of carers' psychological needs. Adv Psychiatr Treat 2003;9(1):45-53. https://doi.org/10.1192/apt.9.1.45.

15. Gusi N et al (2009) Health-related quality of life and fitness of the caregiver of patient with dementia. Med Sci Sports Exerc, 41:1182-1187. https://doi. org/10.1249/MSS.0b013e3181951314, 6

16. Schulz R, Beach SR. Caregiving as a risk factor for mortality: the caregiver health effects study. JAMA. 1999;282(23):2215-9. https://doi.org/10.1001/ja ma.282.23.2215.

17. Fredman $\mathrm{L}$, et al. Leisure-time exercise and overall physical activity in older women caregivers and non-caregivers from the caregiver-SOF study. Prev Med. 2006:43:226-9.

18. Loi SM, Dow B, Ames D, Moore K, Hill K, Russell M, Lautenschlager N (2014) Physical activity in caregivers: what are the psychological benefits? Arch Gerontol Geriatr, 59 2:204-210. https://doi.org/10.1016/j.a rchger.2014.04.001.

19. Hodges $L$, Humphris GM, Macfarlane G. A meta-analytic investigation of the relationship between the psychological distress of cancer patients and their carers. Soc Sci Med. 2005;60(1):1-12. https://doi.org/10.1016/j. socscimed.2004.04.018

20. Northouse L, Williams AL, Given B, McCorkle R. Psychosocial care for family caregivers of patients with cancer. J Clin Oncol. 2012;30(11):1227-34. https://doi.org/10.1200/JCO.2011.39.5798.

21. Barber FD (2013). Effects of social support on physical activity, self-efficacy, and quality of life in adult cancer survivors and their caregivers. Oncol Nurs Forum, 40 5:481-489. https://doi.org/10.1188/13.ONF.481-489.

22. Kershaw TS, Mood DW, Newth G, Ronis DL, Sanda MG, Vaishampayan U, Northouse LL (2008) Longitudinal analysis of a model to predict quality of 
life in prostate cancer patients and their spouses. Ann Behav Med, 36 2:117128, https://doi.org/10.1007/s12160-008-9058-3.

23. Song L, Northouse LL, Braun TM, Zhang L, Cimprich B, Ronis DL, Mood DW (2011). Assessing longitudinal quality of life in prostate cancer patients and their spouses: a multilevel modeling approach. Qual Life Res, 20 3:371-381, https://doi.org/10.1007/s11136-010-9753-y.

24. Wilkinson AV, Barrera SL, McBride CM, Snyder DC, Sloane R, Meneses KM, Pekmezi D, Kraus WE, Demark-Wahnefried W (2012) Extant health behaviors and uptake of standardized vs tailored health messages among cancer survivors enrolled in the FRESH START trial: a comparison of fighting-spirits vs fatalists. Psychooncology, 21 1:108-113. https://doi.org/10.1002/pon.1870.

25. Craig $\mathrm{CL}$, et al. International physical activity questionnaire: 12-country reliability and validity. Med Sci Sports Exerc. 2003;35(8):1381-95. https://doi. org/10.1249/01.MSS.0000078924.61453.FB.

26. Leung DYP, Lam TH, Chan SSC. Three versions of perceived stress scale: validation in a sample of Chinese cardiac patients who smoke. BMC Public Health. 2010;10(1):513. https://doi.org/10.1186/1471-2458-10-513.

27. Fong $T C T, H o R T H$. Factor analyses of the hospital anxiety and depression scale: a Bayesian structural equation modeling approach. Qual Life Res. 2013:1-7.

28. Ware JE, Kosinski M, Keller SD. A 12-item short-form health survey construction of scales and preliminary tests of reliability and validity. Med Care. 1996;34(3):220-33. https://doi.org/10.1097/00005650-199603000-00003.

29. Lam CLK, Tse EYY, Gandek B. Is the standard SF-12 health survey valid and equivalent for a Chinese population? Qual Life Res. 2005;14(2):539-47. https://doi.org/10.1007/s11136-004-0704-3.

30. Muthén LK, Muthén B. Mplus user's guide. 7th ed. Los Angeles, CA: Muthen \& Muthen; 2013.

31. Enders CK, Bandalos DL. The relative performance of full information maximum likelihood estimation for missing data in structural equation models. Struct Equ Model. 2001;8(3):430-57. https://doi.org/10.1207/S1532 8007SEM0803 5.

32. Peugh JL, DiLillo D, Panuzio J. Analyzing mixed-dyadic data using structural equation models. Struct Equ Model Multidiscip J. 2013;20(2):314-37. https:// doi.org/10.1080/10705511.2013.769395.

33. Hu LT, Bentler PM. Cutoff criteria for fit indexes in covariance structure analysis: conventional criteria versus new alternatives. Struct Equ Model. 1999;6(1):1-55. https://doi.org/10.1080/10705519909540118.

34. Mackinnon DP, Lockwood CM, Williams J. Confidence limits for the indirect effect: distribution of the product and resampling methods. Multivar Behav Res. 2004;39(1):99-128. https://doi.org/10.1207/s15327906mbr3901_4.

35. Ng PP (1981) Social factors contributing to fertility decline, in Social life and development in Hong Kong, A.Y.C. King and R.P.L. Lee, Editors. Chinese University Press: Hong Kong, pp235-254.

36. Bjelland I, Dahl AA, Haug TT, Neckelmann D. The validity of the hospital anxiety and depression scale. An updated literature review. J Psychosom Res. 2002;52(2):69-77. https://doi.org/10.1016/S0022-3999(01)00296-3.

37. Speck RM, Courneya KS, Mâsse LC, Duval S, Schmitz KH (2010) An update of controlled physical activity trials in cancer survivors: a systematic review and meta-analysis. J Cancer Surviv, 4:87-100. https://doi.org/10.1007/s11764-0090110-5, 2.

38. Talaei, et al. Physical activity, sex, and socioeconomic status: a population based study. ARYA Atherosclerosis. 2013;9(1):51-60.

39. Motl RW, Snook EM (2008) Physical activity, self-efficacy, and quality of life in multiple sclerosis. Ann Behav Med, 35:111-115. https://doi.org/https://doi. org/10.1007/s12160-007-9006-7, 1

40. Mazanec SR, Sattar A, Delaney CP, Daly BJ (2016) Activation for health management in colorectal cancer survivors and their family caregivers. West J Nurs Res, 38:325-344. https://doi.org/10.1177/0193945915604055, 3

41. Motl RW, McAuley E, Wynn D, Sandroff B, Suh Y (2013) Physical activity, selfefficacy, and health-related quality of life in persons with multiple sclerosis: analysis of associations between individual-level changes over one year. Qual Life Res, 22 2:253-261. https://doi.org/10.1007/s11136-012-0149-z.

42. LeMasters TJ et al (2014) Health behaviors among breast, prostate, and colorectal cancer survivors: a US population-based case-control study, with comparisons by cancer type and gender. J Cancer Surviv, 8 3:336-348. https://doi.org/10.1007/s11764-014-0347-5

\section{Publisher's Note}

Springer Nature remains neutral with regard to jurisdictional claims in published maps and institutional affiliations.

\section{Submit your manuscript to a SpringerOpen ${ }^{\circ}$ journal and benefit from:}

- Convenient online submission

- Rigorous peer review

- Open access: articles freely available online

- High visibility within the field

- Retaining the copyright to your article

Submit your next manuscript at $\boldsymbol{\nabla}$ springeropen.com 